Artículo Original

\title{
Aceites esenciales para el control de Sitophilus zeamais Motschulsky (Coleoptera: Curculionidae) y efecto sobre la calidad del grano de maíz Zea mays Linnaeus (Poales: Poaceae)
}

Essential oils for the control of Sitophilus zeamais Motschulsky (Coleoptera: Curculionidae) and effect on the quality of the corn grain Zea mays Linnaeus (Poales: Poaceae)

\author{
Francyelli Regina Costa-Becheleni ${ }^{1}{ }^{(\mathbb{D})}$, Carmen Lizette Del Toro-Sánchez ${ }^{1}{ }^{\mathbb{D}}$, Francisco Javier \\ Wong-Corral $^{1}{ }^{\mathbb{D}}$, María Del Refugio Robles-Burgueño ${ }^{2}{ }^{\mathbb{D}}$, José Luis Cárdenas-López ${ }^{1}$ y \\ Jesús Borboa-Flores ${ }^{1}$
}

\begin{abstract}
${ }^{1}$ Departamento de Investigación y Posgrado en Alimentos, Universidad de Sonora. Blvd. Luis Encinas y Rosales s/n, Col. Centro, 83000, Hermosillo, Sonora, México. 捣 jesus.borboa@unison.mx

${ }^{2}$ Centro de Investigación en Alimentación y Desarrollo. Carretera Gustavo Enrique Astiazarán Rosas, 46, 83304, Hermosillo, Sonora, México.
\end{abstract}

\section{ZooBank: urn:lsid:zoobank.org:pub:6A80FBF2-8A2B-412F-B2EE-2E0710554546 https: / / doi.org/10.35249/rche.46.4.20.10}

Resumen. Con la finalidad de buscar alternativas naturales para el control del gorgojo Sitophilus zeamais Motschulsky, se evaluó in vitro el efecto de los aceites esenciales de niaouli (Melaleuca quinquenervia), eucalipto (Eucalyptus globulus) y orégano (Origanum vulgare) sobre la mortalidad, emergencia y repelencia de $S$. zeamais, así como su efecto en la calidad del grano de maíz durante su almacenamiento (germinación, cantidad de fenoles, flavonoides y capacidad antioxidante). La técnica utilizada para los bioensayos fue la de saturación de atmósferas, en volúmenes de 100, 200 y $300 \mu \mathrm{L}$ de los aceites, teniendo como sustrato maíz blanco nacional. Los tres aceites presentaron actividad insecticida del 100\% a las $72 \mathrm{hrs}$. La emergencia de insectos adultos $\left(\mathrm{F}_{1}\right)$ fue nula en todos los tratamientos. La repelencia se observó en todos los tratamientos a las 24, 48 y 72 hrs. La germinación no se vió afectada en volúmenes de $100 \mu \mathrm{L}$. El contenido de fenoles y flavonoides totales fueron mayores tanto en el aceite de orégano (94,68 mg EAG/g y 0,27 mg EQ/g respectivamente) como en el tratamiento maíz con orégano (16,09 mg EAG/g y 0,18 mg EQ/g respectivamente). Similar comportamiento se observó en la capacidad antioxidante tanto en DPPH como en ABTS presentando rangos de 76,31 a 93,73\% de inhibición. Se concluye que los tres aceites esenciales presentan potencial actividad para el control de $S$. zeamais mejorando la calidad del grano de maíz.

Palabras clave: Antioxidantes, bioinsecticida, biocontrol, metabolitos secundarios.

Abstract. In order to find natural alternatives for the control of the weevil Sitophilus zeamais Motschulsky, the effect of niaouli (Melaleuca quinquenervia), eucalyptus (Eucalyptus globulus) and oregano (Origanum vulgare) essential oils were studied on mortality, emergence and repellency of $S$. zeamais, as well as their effect on the grain quality of corn during storage (germination, amount of phenols, flavonoids and antioxidant capacity). The technique used for the bioassays was saturation of atmospheres, in volumes of 100, 200 and $300 \mu \mathrm{L}$, having national white corn as substrate. The three oils showed 100\% insecticidal activity at $72 \mathrm{hrs}$. The emergence of adult insects $\left(\mathrm{F}_{1}\right)$ was null in all treatments. Repellency was observed in all treatments at 24, 48 and 72 hrs. Germination was

Recibido 29 Julio 2020 / Aceptado 6 Noviembre 2020 / Publicado online 23 Diciembre 2020 Editor Responsable: José Mondaca E. 
not affected in volumes of $100 \mu \mathrm{L}$. The content of total phenols and flavonoids were higher both in the oregano oil (94.68 mg GAE/g and $0.27 \mathrm{mg} \mathrm{QE} / \mathrm{g}$ respectively) and in the corn treatment with oregano (16.09 mg GAE/g and $0.18 \mathrm{mg}$ QE/g respectively). Similar behavior was observed in the antioxidant capacity in both DPPH and ABTS, presenting ranges from 76.31 to $93.73 \%$ inhibition. It is concluded that the three essential oils have potential for the control of $S$. zeamais, improving the quality of the corn grain.

Key words: Antioxidants, biocontrol, bioinsecticide, secondary metabolites.

\section{Introducción}

La importancia de conservar los granos almacenados requiere cuidados, pues debido al crecimiento de la población se busca asegurar el abastecimiento de alimentos con excelente calidad, que no sufran daños por la acción de plagas, enfermedades o del medio ambiente, evitando pérdidas en su peso o reducción en su calidad (FAO 2010). Adicionalmente, los agricultores deben regular los factores físicos como temperatura, humedad, oxígeno y las condiciones del grano como la limpieza, aireación y monitoreo (Blanco et al. 2016) para no afectar el crecimiento y la producción, controlando o minimizando esos daños y haciendo importante el estudio para disminuir las pérdidas en postcosecha (Carvalho et al. 2017). Un buen sistema de almacenamiento permite disminuir las mermas ocasionadas por plagas y enfermedades, reduciendo así los costos económicos, ambientales y sociales que estos generan (Ortiz et al. 2012). La finalidad del almacenamiento del grano es conservar las condiciones adecuadas, con el propósito de preservar la calidad hasta a su consumo (Santos y Chavaglia 2017). Uno de los grandes problemas que tienen los productores durante el almacenamiento de granos, es su elevada pérdida económica ocasionada por la acción de insectos plaga, ya que estos interfieren en la calidad y afectan la producción alimentaria (Lima y Cardoso 2013; Antunes y Dionello 2017). El gorgojo del maíz Sitophilus zeamais Motschulsky, 1855 (Coleoptera: Curculionidae) destaca en importancia económica como la principal plaga de los cultivos de maíz, debido a los daños que provocan en sus productos (Elias et al. 2009). Sitophilus zeamais se considera también el insecto más importante de las plagas de postcosecha del maíz (Rees 1996; Assis et al. 2014), el cual se estima que en México puede causar mermas del 4 al 25\% (Ortiz et al. 2015). Es una plaga primaria, en que las larvas y adultos se alimentan del interior del grano (Lagunes 1994). Está ampliamente distribuida en regiones tropicales y subtropicales del mundo (Assis et al. 2014). El control químico es el método más utilizado para proteger los granos almacenados del ataque de este insecto (Florão et al. 2004; Docampo et al. 2014). Sin embargo, debido a la composición química y toxicidad de los productos utilizados, presenta desventajas como contaminación de los granos por residuos, resistencia de los insectos, riesgos graves al medio ambiente, daños a la salud, alto costo de los productos, necesidad de equipos de aplicación y mano de obra entrenada (Finkler 2011; Campanini et al. 2012). Esta situación, ha originado la búsqueda y adopción de nuevas alternativas de control que permitan reducir el uso indiscriminado de agrotóxicos, que sean efectivos y a la vez no presente efectos perjudiciales (Pessoa et al. 2014). Entre las opciones se encuentran los productos de origen natural derivados de plantas como son los aceites esenciales. Se definen como mezclas complejas de fracciones volátiles y sustancias aromáticas líquidas, con más de cien compuestos químicos orgánicos provenientes de la familia de los terpenoides que forman parte del metabolismo secundario de las plantas y que se producen de manera natural para la defensa contra insectos y otros microrganismos (Usano et al. 2014). Por lo anterior, el presente estudio es con el propósito de obtener información y contribuir en la búsqueda de productos naturales que puedan controlar a $S$. zeamais sin afectar la 
germinación y aumentando la calidad del grano. Por lo tanto, se evaluaron los aceites esenciales de niaouli (Melaleuca quinquenervia (Cav. S. T. Blake), eucalipto (Eucalyptus globulus Labill.) y orégano (Origanum vulgare Linnaeus) como método alternativo de control de plagas de insectos, determinando la mortalidad, la emergencia y el índice de repelencia de $S$. zeamais con cada uno de los aceites esenciales y evaluando la germinación y capacidad antioxidante del grano de maíz almacenado.

\section{Materiales y Métodos}

La investigación se realizó en el Laboratorio de Entomología del Departamento de Investigación y Posgrado en Alimentos de la Universidad de Sonora, Campus Centro, Hermosillo, Sonora México.

Insectos. Los ejemplares de $S$. zeamais se recolectaron en almacenes de cereales infestados, en la región del estado de Sonora, coordenadas 95'21" N, 11057'40" O. La identificación de los adultos se realizó mediante las claves taxonómicas de Gorham (1999), y se mantuvieron en frascos de cristal de 1 L con maíz (Zea mays L.) blanco nacional como sustrato alimenticio, a $27 \pm 1{ }^{\circ} \mathrm{C}$, con $70 \pm 5 \%$ HR y 12:12 hrs de luz: oscuridad en cámara de incubación.

Aceites. Los aceites esenciales utilizados fueron niaouli (M. quinquenervia), eucalipto (E. globulus) y orégano (O. vulgare), adquiridos del comercio local con grado de pureza del $99 \%$.

\section{Bioensayo de actividad volátil (toxicidad)}

La toxicidad por efecto fumigante se avaluó de acuerdo con la metodología de ReyesGuzmán et al. (2012). En la parte interna de la tapa de vasos de plástico de $200 \mathrm{~mL}$, se colocó una esponja de poliuretano de 0,5 x 0,5 cm impregnada con 100, 200 y $300 \mu \mathrm{L}$ de los aceites esenciales de M. quinquenervia, E. globulus y O. vulgare, más un testigo que consistió en la esponja sin impregnación de aceites. En los envases se colocaron 20 g de grano de maíz y 10 adultos de $S$. zeamais. La evaluación de cada aceite esencial fue por triplicado. Se contaron insectos vivos y muertos a las 24, 48 y 72 hrs. El experimento se realizó a temperatura de $27 \pm 2{ }^{\circ} \mathrm{C}, 70 \% \mathrm{HR}$ y un fotoperiodo de $12 \mathrm{hrs}$ luz y $12 \mathrm{hrs}$ de oscuridad. Se consideró como insecto muerto a todos los que no mostraron movimientos ante la punción con aguja de disección entomológica. Los resultados de mortalidad se calcularon mediante la fórmula de Abbott (1925).

\section{Emergencia de insectos adultos $\left(\mathrm{F}_{1}\right)$}

Una vez terminada las 72 hrs de evaluación de mortalidad se retiraron todos los insectos adultos de cada uno de los frascos y estos conteniendo sólo maíz se regresaron a las cámaras de incubación por 45 días más. Al final de este período se evaluó la emergencia de insectos adultos $\left(\mathrm{F}_{1}\right)$ tomando como $100 \%$ la $\mathrm{F}_{1}$ del testigo en los tiempos de 24, 48 y 72 horas.

\section{Bioensayo de repelencia}

La repelencia se evaluó siguiendo la metodología de Mazzonetto (2002). La unidad experimental consistió en 2 vasos de plástico de $200 \mathrm{~mL}$, conectados lateralmente por un tubo de plástico de $5 \mathrm{~cm}$ de largo y 0,5 de diámetro, con una perforación en la parte 
media del tubo para introducir a los insectos. En la parte interna de la tapa del vaso se colocaron esponjas de 0,5 x 0,5 para aplicar el aceite esencial. En cada uno de los vasos se colocaron 25 g de maíz y 10 insectos adultos de $S$. zeamais con 48 horas de edad. Las esponjas fueron impregnadas de 100, 200 y $300 \mu \mathrm{L}$ de los aceites esenciales. Los vasos de la unidad experimental se sellaron y se mantuvieron en cámara de incubación a $27^{\circ} \mathrm{C}$ y $70 \%$ HR. En otros tres vasos se ubicó el testigo con sólo granos de maíz sin el aceite esencial. Después de 24, 48 y 72 hrs se contabilizó el número de insectos por vaso. Cada tratamiento tuvo tres repeticiones y con los adultos obtenidos se calculó el índice de repelencia (IR) que clasifica el tratamiento como neutro si el IR $=1$, atrayente si el IR $>1$ y repelente si el IR $<1$.

\section{Determinación de la germinación}

La prueba de germinación se realizó bajo condiciones controladas de laboratorio según las normas ISTA (2016), para análisis de semillas de cereales, utilizando como sustrato charolas de germinación, papel kim-Pak y papel estraza. En frascos de cristal de 1 L se colocaron $200 \mathrm{~g}$ de semilla de maíz. En la parte interna de las tapas se colocó una esponja de poliuretano de 4,0 x 3,0 cm, donde se aplicaron los aceites esenciales con 100, 200 y $300 \mu \mathrm{L}$, siendo el control sin aceite esencial. Las tapas se sellaron con papel Parafilm y se incubaron a $27^{\circ} \mathrm{C}$ y $70 \% \mathrm{HR}$ con fotoperiodo de $12 \mathrm{hrs}$ luz: $12 \mathrm{hrs}$ oscuridad durante 31 días. Para germinar, se seleccionaron 300 semillas de cada uno de los frascos, siendo distribuidos en bandejas de germinación de 50 x $40 \mathrm{~cm}$ con 100 cavidades por triplicado. Las bandejas se llevaron a una germinadora automática de doble cámara marca Seedburo, modelo 1000 FAAT, acondicionada a temperatura de $25^{\circ} \mathrm{C}$ y $100 \%$ HR durante 8 días. Los conteos de germinación se realizaron evaluando el crecimiento de plántulas normales, plántulas anormales y semillas no germinadas.

\section{Obtención de extractos de maíz para la determinación de la actividad biológica}

Los extractos de las muestras de maíz se obtuvieron de acuerdo con la metodología establecida por Morales et al. (2015) con modificaciones. Se tomaron 100 g de maíz de cada una de las muestras y se sometieron a liofilización. Se molió la muestra y se utilizó un tamiz para obtener un tamaño de partícula de 0,425 mm. Posteriormente, se pesaron $5 \mathrm{~g}$ de cada una de las harinas por duplicado añadiendo $20 \mathrm{~mL}$ de metanol. Las suspensiones obtenidas se homogeneizaron siendo sometidas a sonicación por $30 \mathrm{~min}$ a $68^{\circ} \mathrm{C}$. Después se sometieron a agitación $(200 \mathrm{rpm})$ durante $12 \mathrm{hrs}$ protegidas de la luz. Posteriormente se centrifugaron a $4.000 \mathrm{rpm}$ a $4^{\circ} \mathrm{C}$ por $15 \mathrm{~min}$. Los sobrenadantes se concentraron utilizando un rotavapor a $65 \mathrm{rpm}$ a $40^{\circ} \mathrm{C}$. Finalmente, se suspendieron en $2 \mathrm{~mL}$ de metanol obteniendo una concentración final de $0,02 \mathrm{~g} / \mathrm{mL}$ para la cuantificación del contenido de fenoles y flavonoides totales y actividad antioxidante por DPPH y ABTS.

\section{Cuantificación de fenoles totales}

Para determinar los fenoles totales de los extractos de maíz y de los aceites esenciales, se utilizó la técnica de Folin-Ciocalteu de acuerdo con Eldeen et al. (2011). Para la medición de las muestras se utilizó una placa de 96 pocillos, colocando $10 \mu \mathrm{L}$ de muestra $+25 \mu \mathrm{L}$ de solución de Folin 1N. Se dejó en reposo durante 5 minutos. Posteriormente a todas las muestras se le agregaron $25 \mu \mathrm{L}$ de carbonato de sodio al $20 \%$ y $140 \mu \mathrm{L}$ de agua destilada para llegar al volumen final de $200 \mu \mathrm{L}$. Después de $30 \mathrm{~min}$ de reposo, se determinó la absorbancia a $760 \mathrm{~nm}$. Los resultados se expresaron como miligramos de equivalentes de ácido gálico por gramo de muestra (mgEAG/g). Todas las muestras se evaluaron por triplicado. 


\section{Cuantificación de flavonoides totales}

Para determinar los flavonoides totales se utilizó la metodología de acuerdo con Venu et al. (2012). En una placa de 96 pocillos se colocaron $80 \mu \mathrm{L}$ de muestra $+80 \mu \mathrm{L}$ de solución etanólica de tricloruro de aluminio $(20 \mathrm{~g} / \mathrm{L})$. Se dejó reposar durante 1 hora en oscuridad y se determinó la absorbancia a $415 \mathrm{~nm}$. Los resultados se expresaron como miligramos de equivalentes de quercetina por gramo de muestra (mgEQ/g). Todas las muestras se evaluaron por triplicado.

\section{Cuantificación de antioxidantes por el método de DPPH y ABTS}

Para determinar la actividad antioxidante por la técnica de DPPH (2,2-difenil-1picrilhydrazil) se utilizó la metodología de Molyneux (2004). En una placa de 96 pocillos se colocó $220 \mu \mathrm{L}$ radical DPPH $(0,05 \mathrm{mg} / \mathrm{mL})+20 \mu \mathrm{L}$ de muestra. El control tenía $220 \mu \mathrm{L}$ radical DPPH $+20 \mu \mathrm{L}$ de metanol. Se dejó reposar durante 30 min para posteriormente medir la absorbancia a $515 \mathrm{~nm}$. Los resultados se reportaron como \% de inhibición de acuerdo con la siguiente ecuación:

$$
\% \text { de inhibición }=\frac{\text { absorbancia control-absorbancia muestra }}{\text { absorbancia control }} \times 100
$$

Para determinar la actividad antioxidante por la técnica de ABTS (2,2'-Azinobis-3etil-benzotiazolina-6-ácido-sulfónico) se utilizó la metodología de Manach et al. (2004). Posterior a la formación del radical ABTS •+ durante $16 \mathrm{hrs}$ en contacto con persulfato de potasio como lo indica la referencia, se ajustó a una absorbancia de 0,7 $\pm 0,02$ a $734 \mathrm{~nm}$. Posteriormente, en una placa de 96 pocillos se colocaron $270 \mu \mathrm{L}$ de radical ABTS ${ }^{\bullet+}$ con $20 \mu \mathrm{L}$ muestra. El control fue $270 \mu \mathrm{L}$ radical ABTS con $20 \mu \mathrm{L}$ de metanol. Se dejó reposar durante $30 \mathrm{~min}$ y posteriormente se midió la absorbancia a $734 \mathrm{~nm}$. Los resultados se reportaron como \% de inhibición utilizando la misma fórmula que en DPPH.

\section{Diseño experimental y análisis estadístico}

Para las pruebas entomológicas se utilizó un diseño factorial para un insecto y tres diferentes aceites esenciales a cuatro volúmenes de 0, 100, 200 y $300 \mu \mathrm{L}$, a tres tiempos de exposición de 24, 48 y 72 hrs con 3 repeticiones. Las variables respuesta medidas fueron el porcentaje de mortalidad, emergencia y repelencia. Para las pruebas de calidad se utilizó un diseño factorial para un tipo de maíz y tres diferentes aceites esenciales a cuatro volúmenes de $0,100,200$ y $300 \mu \mathrm{L}$, a un tiempo de exposición de 31 días con 3 repeticiones. Las variables respuesta medidas fueron el porcentaje de germinación, fenoles totales, flavonoides totales, DPPH y ABTS. Los resultados obtenidos de las pruebas entomológicas y de las pruebas de calidad fueron analizados por estadística descriptiva y análisis de varianza ANOVA. Para el análisis de los datos se utilizó el programa estadístico de cómputo InfoStat versión 2018 mediante la prueba de comparaciones múltiples de Tukey, considerando el intervalo de confianza del 95\% $(\mathrm{p}<0,05)$.

\section{Resultados y Discusión}

\section{Porcentaje de mortalidad}

Los aceites esenciales de E. globulus y M. quinquenervia fueron los más eficientes en el control de $S$. zeamais, causando mortalidad de $100 \%$ en todos las concentraciones y tiempos evaluados $(\mathrm{p}<0,05)$ (Tabla 1). Las concentraciones de O. vulgare de 100, 200 y $300 \mu \mathrm{L}(\mathrm{v} / \mathrm{p})$ 
en 24 hrs fluctuando en 30, 40 y 50\%, por lo cual quedan fuera del umbral establecido para el uso de cualquier producto de origen botánico que sea favorable en provocar una tasa de mortalidad superior al 50\% (Lagunes 1994). Sin embargo, el mismo aceite en 48 y 72 hrs en todas las concentraciones superaron el 90\% de mortalidad de S. zeamais. Estos resultados concuerdan con Martínez et al. (2015), quienes utilizaron aceite esencial de L. palmeri contra adultos S. zeamais y P. truncatus en dosis $1000 \mu \mathrm{l} /$ litro, indicó que la mortalidad en 24 hrs fue de $78 \%$ y en 48 hrs de $100 \%$ para S. zeamais, y para P. truncatus en 72 hrs fue $92 \%$, indicando que, conforme aumentó la concentración del aceite y el tiempo de exposición mayor fue la mortalidad. Reyes-Guzmán et al. (2012), reportaron que en adultos de Ryhzopertha dominica el aceite de E. globulus causó 100\% de mortalidad en 24 hrs en volúmenes de 10 y $15 \mu \mathrm{L}$, estos resultados difieren del actual en el volumen utilizado, sin embargo, existe similitud en el tiempo. Lo anterior demuestra que la acción fumigante de los aceites esenciales y sus principales constituyentes varía con la especie de insecto, la concentración del aceite y el tiempo de exposición, por lo que muestran alta toxicidad específica, siendo dependientes de la dosis y del tiempo de exposición (Ogendo et al. 2008).

Tabla 1. Porcentaje de mortalidad de S. zeamais en maíz expuestos a aceites senciales ${ }^{1,2}$.

\begin{tabular}{|c|c|c|c|c|c|c|c|c|c|}
\hline \multicolumn{4}{|c|}{ Niaouli (M. quinquenervia) } & \multicolumn{3}{|c|}{ Eucalipto (E. globulus) } & \multicolumn{3}{|c|}{ Orégano (O. vulgare) } \\
\hline \multicolumn{4}{|c|}{ Tiempo (h) } & \multicolumn{3}{|c|}{ Tiempo (h) } & \multicolumn{3}{|c|}{ Tiempo (h) } \\
\hline $\begin{array}{l}\text { *Vol. } \\
(\mu \mathrm{L})\end{array}$ & 24 & 48 & 72 & 24 & 48 & 72 & 24 & 48 & 72 \\
\hline 0 & $0,0 \pm 0,0$ & $0,0 \pm 0,0$ & $0,0 \pm 0,0$ & $0,0 \pm 0,0$ & $0,0 \pm 0,0$ & $0,0 \pm 0,0$ & $0,0 \pm 0,0$ & $0,0 \pm 0,0$ & $0,0 \pm 0,0$ \\
\hline 100 & $100,0^{\mathrm{a}} \pm 0,0$ & $100,0^{\mathrm{a}} \pm 0,0$ & $100,0^{\mathrm{a}} \pm 0,0$ & $100,0 \mathrm{a} \pm 0,0$ & $100,0^{\mathrm{a}} \pm 0,0$ & $100,0^{\mathrm{a}} \pm 0,0$ & $030,0^{\mathrm{a}} \pm 1,0$ & $90,0^{b} \pm 0,0$ & $100,0^{c} \pm 0,0$ \\
\hline 200 & $100,0^{\mathrm{a}} \pm 0,0$ & $100,0^{\mathrm{a}} \pm 0,0$ & $100,0^{\mathrm{a}} \pm 0,0$ & $100,0^{\mathrm{a}} \pm 0,0$ & $100,0^{\mathrm{a}} \pm 0,0$ & $100,0^{\mathrm{a}} \pm 0,0$ & $040,0^{\mathrm{a}} \pm 1,0$ & $96,0^{\mathrm{bc}} \pm 0,5$ & $100,0^{c} \pm 0,0$ \\
\hline 300 & $100,0^{\mathrm{a}} \pm 0,0$ & $100,0^{\mathrm{a}} \pm 0,0$ & $100,0^{\mathrm{a}} \pm 0,0$ & $100,0^{\mathrm{a}} \pm 0,0$ & $100,0^{\mathrm{a}} \pm 0,0$ & $100,0^{\mathrm{a}} \pm 0,0$ & $050,0^{\mathrm{a}} \pm 1,0$ & $100,0^{c} \pm 0,0$ & $100,0 \subset \pm 0,0$ \\
\hline
\end{tabular}

"Volúmenes en $\mu \mathrm{L}$ de aceite esencial que fueron expuestos los granos de maíz y el insecto. ${ }^{1}$ Los valores son el promedio de tres réplicas \pm desviación estándar. ${ }^{2}$ Las letras diferentes entre líneas indican diferencia significativa $(p<0,05)$. Análisis de datos obtenidos del paquete estadístico Infostat versión 2018.

\section{Emergencia de insectos adultos (F1)}

Todos los tratamientos se consideran como prometedores ya que redujeron menos del $50 \%$ de la $\mathrm{F}_{1}$ de S. zeamais, resultados acordes con Silva et al. (2005). No se observó emergencia en ningún tratamiento, solamente en los controles $(p<0,05)$ (Tabla 2). Estos resultados concuerdan con los de Langsi et al. (2018), quienes reportaron que a concentración de $200 \mu \mathrm{L} \mathrm{kg}^{-1}$ de aceite esencial de Ch. ambrosioides obtuvieron una inhibición de $100 \%$ de la $\mathrm{F}_{1}$ de S. zeamais. Reyes-Guzmán et al. (2012), lograron con el aceite esencial de eucalipto la inhibición de la progenie $\mathrm{F}_{1}$ de Rhyzopertha dominica (Fabricius) en dosis de 10 y $15 \mu \mathrm{L}$ y con un tiempo de exposición 72 hrs, indicando que las dosis influyen directamente, teniendo mayor efecto en la oviposición. Çalmaşur et al. (2006), estudiaron el vapor del aceite esencial de orégano utilizando dosis de 2, 4, 6 y $8 \mu \mathrm{L} / \mathrm{L}$, durante las 24, 48, 96 y 120 hrs para el control de ninfas y adultos de Tetranychus urticae Koch, siendo más efectivo el control con la dosis más elevada y al mayor tiempo de exposición. El factor determinante para que no se observara emergencia fue la eliminación total de la población de adultos de S. zeamais antes de la oviposición, y en consecuencia suprimida la oviposición eliminando los huevos y larvas debido a la toxicidad de los compuestos volátiles del aceite esencial. 
Tabla 2. Porcentaje de emergencia de S. zeamais en maíz expuestos a aceites esenciales ${ }^{1,2}$.

\begin{tabular}{|c|c|c|c|c|c|c|c|c|c|}
\hline \multicolumn{4}{|c|}{ Niaouli (M. quinquenervia) } & \multicolumn{3}{|c|}{ Eucalipto (E.globulus) } & \multicolumn{3}{|c|}{ Orégano (O. vulgare) } \\
\hline \multicolumn{4}{|c|}{ Tiempo (h) } & \multicolumn{3}{|c|}{ Tiempo (h) } & \multicolumn{3}{|c|}{ Tiempo (h) } \\
\hline $\begin{array}{l}{ }^{*} \text { Vol. } \\
(\mu \mathrm{L})\end{array}$ & 24 & 48 & 72 & 24 & 48 & 72 & 24 & 48 & $\overline{72}$ \\
\hline 0 & $43,0^{\mathrm{a}} \pm 2,3$ & $67,0^{\mathrm{b}} \pm 4,1$ & $17,0^{c} \pm 1,1$ & $63,0^{\mathrm{a}} \pm 5,0$ & $30,0^{\mathrm{b}} \pm 2,5$ & $60,0^{\mathrm{a}} \pm 3,0$ & $30,0^{\mathrm{a}} \pm 1,7$ & $70,0^{\mathrm{b}} \pm 1,3$ & $30,0^{\mathrm{a}} \pm 1,0$ \\
\hline 100 & $0,0 \pm 0,0$ & $0,0 \pm 0,0$ & $0,0 \pm 0,0$ & $0,0 \pm 0,0$ & $0,0 \pm 0,0$ & $0,0 \pm 0,0$ & $0,0 \pm 0,0$ & $0,0 \pm 0,0$ & $0,0 \pm 0,0$ \\
\hline 200 & $0,0 \pm 0,0$ & $0,0 \pm 0,0$ & $0,0 \pm 0,0$ & $0,0 \pm 0,0$ & $0,0 \pm 0,0$ & $0,0 \pm 0,0$ & $0,0 \pm 0,0$ & $0,0 \pm 0,0$ & $0,0 \pm 0,0$ \\
\hline 300 & $0,0 \pm 0,0$ & $0,0 \pm 0,0$ & $0,0 \pm 0,0$ & $0,0 \pm 0,0$ & $0,0 \pm 0,0$ & $0,0 \pm 0,0$ & $0,0 \pm 0,0$ & $0,0 \pm 0,0$ & $0,0 \pm 0,0$ \\
\hline
\end{tabular}

Volúmenes en $\mu \mathrm{L}$ de aceite esencial que fueron expuestos los granos de maíz y el insecto. ${ }^{1}$ Los valores son el promedio de tres réplicas \pm desviación estándar. ${ }^{2}$ Letras diferentes en línea indican diferencia significativa $(\mathrm{p}<0,05)$. Análisis de datos obtenidos del paquete estadístico Infostat versión 2018.

\section{Índice de repelencia (IR)}

Todos los tratamientos mostraron IR menores a 1, lo cual los clasifica como repelentes. La repelencia aumentó en el tratamiento con $300 \mu \mathrm{L}$ de aceite esencial con 72 hrs de exposición siendo de 0,4 y 0,5 para cada uno de los tratamientos $(\mathrm{p}<0,05)$ (Tabla 3). Estos resultados coinciden con los de Norambuena et al. (2016), quienes señalaron que el aceite esencial de Laureliopsis philippiana (Looser) Schodde en dosis de 0,25, 0,5, 1,0, 2,0 y 4,0\% (v/v) a las 24 hrs contra Sitophilus zeamais (Most.), Sitophilus oryzae (L.) y Sitophilus granarius (L.), son repelentes, con el IR $<1$. Para $S$. zeamais los IR observados en las dosis de 0,25 y 0,5\% fueron de 0,8 y 0,9, las dosis más altas de 2,0 y 4,0\% presentaron IR cercanos a cero $(0,3$ y $0,2)$, mencionando que las dosis mayores tienen un índice comparativamente menor lo que implica un mayor efecto repelente. Bustos et al. (2017), que estudiaron los aceites esenciales de Peumus boldus, Laurelia sempervirens y Laureliopsis philippiana en dosis de 0,5, 1,0, 2,0, 4,0 y $8,0 \%(\mathrm{v} / \mathrm{v})$ a las $24 \mathrm{hrs}$ contra $S$. zeamais reportaron actividad repelente entre ambos tratamientos, con el IR $<1$, indicando IR más bajos en las dosis más altas de 4,0 y 8,0\%. Estos porcentajes concuerdan con la presente investigación ya que los valores de repelencia en 24 hrs fueron $<1$. Estos resultados son de gran utilidad, para considerar a los aceites de M. quinquenervia, E. globulus y O. vulgare como una alternativa altamente repelente para el control de S. zeamais.

Tabla 3. Índices de repelencia de S. zeamais en maíz expuestos a aceites esenciales ${ }^{1,2}$.

\begin{tabular}{|c|c|c|c|c|c|c|c|c|c|}
\hline \multicolumn{4}{|c|}{ Niaouli (M. quinquenervia) } & \multicolumn{3}{|c|}{ Eucalipto (E.globulus) } & \multicolumn{3}{|c|}{ Orégano (O. vulgare) } \\
\hline \multicolumn{4}{|c|}{ Tiempo (h) } & \multicolumn{3}{|c|}{ Tiempo (h) } & \multicolumn{3}{|c|}{ Tiempo (h) } \\
\hline $\begin{array}{l}\text { *Vol. } \\
(\mu \mathrm{L})\end{array}$ & 24 & 48 & 72 & 24 & 48 & 72 & 24 & 48 & 72 \\
\hline 0 & $1,3^{\mathrm{a}} \pm 0,3$ & $1,2^{\mathrm{a}} \pm 0,5$ & $1,2^{\mathrm{a}} \pm 0,2$ & $1,0^{\complement} \pm 0,1$ & $1,2^{a} \pm 0,3$ & $1,2^{a} \pm 0,5$ & $1,6^{\mathrm{b}} \pm 0,2$ & $1,0^{c} \pm 0,5$ & $1,3^{\underline{a}} \pm 0,4$ \\
\hline 100 & $0,8^{\mathrm{f}} \pm 0,3$ & $0,8^{f} \pm 0,3$ & $50,8^{f} \pm 0,2$ & $0,9^{g} \pm 0,1$ & $0,9 \mathrm{~g} \pm 0,3$ & $0,8^{\mathrm{f}} \pm 0,5$ & $0,9^{g} \pm 0,2$ & $0,9 \mathrm{~g} \pm 0,5$ & $0,9 \mathrm{~g} \pm 0,4$ \\
\hline 0 & $1,1^{\mathrm{a}} \pm 0,3$ & $1,0^{c} \pm 0,3$ & $1,4^{\mathrm{c}} \pm 0,21$ & $2,0^{a} \pm 0,3$ & $1,0^{\mathrm{c}} \pm 0,3$ & $1,2^{\underline{a}} \pm 0,2$ & $1,1^{\underline{a}} \pm 0,4$ & $1,0^{c} \pm 0,2$ & $1,0^{\mathrm{c}} \pm 0,5$ \\
\hline 200 & $0,7^{\mathrm{ef}} \pm 0,3$ & $0,8^{f} \pm 0,3$ & $0,6^{\text {de }} \pm 0,2$ & $0,8^{f} \pm 0,3$ & $0,7^{\mathrm{ef}} \pm 0,3$ & $0,7^{\mathrm{ef}} \pm 0,2$ & $0,8^{\mathrm{f}} \pm 0,4$ & $0,9 g \pm 0,2$ & $0,6^{\mathrm{de}} \pm 0,5$ \\
\hline 0 & $1,2^{\underline{a}} \pm 0,3$ & $1,3^{\mathrm{a}} \pm 0,4$ & $1,6^{\mathrm{b}} \pm 0,2$ & $1,2^{\underline{a}} \pm 0,5$ & $1,2^{\mathrm{a}} \pm 0,3$ & $1,0^{c} \pm 0,5$ & $1,0^{c} \pm 0,3$ & $1,2^{\underline{a}} \pm 0,2$ & $1,4^{\mathrm{c}} \pm 0,2$ \\
\hline 300 & $0,6^{\mathrm{de}} \pm 0,3$ & $0,6^{\mathrm{de}} \pm 0,4$ & $0,4^{\mathrm{d}} \pm 0,2$ & $0,8^{f} \pm 0,3$ & $0,7^{\mathrm{ef}} \pm 0,3$ & $0,5^{\mathrm{h}} \pm 0,5$ & $0,8^{\mathrm{f}} \pm 0,3$ & $0,8^{\mathrm{f}} \pm 0,2$ & $0,5^{\mathrm{h}} \pm 0,2$ \\
\hline
\end{tabular}

*Volúmenes en $\mu \mathrm{L}$ de aceite esencial que fueron expuestos los granos de maíz y el insecto. ${ }^{1}$ Los valores son el promedio de tres réplicas \pm desviación estándar. ${ }^{2}$ Letras diferentes en columna indican diferencia significativa $(\mathrm{p}<0,05)$. Análisis de datos obtenidos del paquete estadístico Infostat versión 2018. IR calculados según descrito por Mazzoneto (2002). IR=1 tratamiento neutro IR $>1$ tratamiento atrayente $I R<1$ tratamiento repelente. 


\section{Porcentaje de germinación}

La menor germinación se registró con el tratamiento de $300 \mu \mathrm{L}$ con valores de $65,4,78,7$ y 65,7\% en los tratamientos de M. quinquenervia, E. globulus y O. vulgare respectivamente, mientras que en $100 \mu \mathrm{L}$, se observó una germinación de 83,7, 81,0 y 81,0\% (Tabla 4). Se encontraron diferencias significativas entre los volúmenes de aceite esencial, lo que indica que a mayor concentración del aceite el porcentaje de germinación fue menor (Tabla 5). González-Guiñez et al. (2016), utilizando aceite esencial de Eucalyptus globulus y Eucalyptus nitens H. Deane y Maiden en concentración de 0,25 a 8,00 (v/v), reporta porcentajes de 95 a 100\% de germinación. Similar al presente trabajo la germinación disminuye conforme aumenta la concentración de aceites esenciales. Reyes-Guzmán et al. (2012), estudiando los granos de trigo con los aceites de Eucalyptus globulus y Eucalyptus camaldulensis Dehnh. reportaron una germinación del 90 al 100\% en dosis de 5, 10 y $15 \mu \mathrm{L}$. Salas (1985) y González (1995) reportaron que la disminución de germinación podría ser consecuencia de la composición de los granos de maíz por presentar mayoritariamente un endosperma harinoso y amiláceo, el cual puede absorber elevadas concentraciones de terpenoides, afectando directamente al embrión y efectos perjudiciales en el procesos fisiológico y metabólico de la semilla. Cabe mencionar, que la germinación obtenida en la presente investigación (menor a 85\%), no cumple con los porcentajes establecidos por los estándares nacionales para ser comercializadas como semilla de alta calidad (SAG 2000).

Tabla 4. Porcentaje de germinación de maíz con los aceites esenciales ${ }^{1,2}$.

\begin{tabular}{|c|c|c|c|c|c|c|c|c|c|}
\hline \multirow[b]{3}{*}{$\begin{array}{l}{ }^{*} \text { Vol. } \\
(\mu \mathrm{L})\end{array}$} & \multicolumn{3}{|c|}{ Niaouli (M. quinquenervia) } & \multicolumn{3}{|c|}{ Eucalipto (E. globulus) } & \multicolumn{3}{|c|}{ Orégano (O. vulgare) } \\
\hline & \multicolumn{3}{|c|}{ Parámetros (plántulas) } & \multicolumn{3}{|c|}{ Parámetros (plántulas) } & \multicolumn{3}{|c|}{ Parámetros (plántulas) } \\
\hline & Normales & Anormal & $\begin{array}{l}\text { es Sin } \\
\text { Germinar }\end{array}$ & Normales & Anormale & $\begin{array}{l}\text { es Sin } \\
\text { Germina }\end{array}$ & $\begin{array}{l}\text { Normales } \\
\text { ir }\end{array}$ & Anormales & $\begin{array}{l}\text { Sin } \\
\text { Germinar }\end{array}$ \\
\hline 0 & $86,4^{a} \pm 5,5$ & $6,0^{b} \pm 3,6$ & $7,7^{0}=$ &, 5 & $3,7 \mathrm{a}=$ & $9,0^{a} \pm 3,0$ & $, 7 \mathrm{a} \pm 3,2$ & 0,5 & 6,0 \\
\hline 100 & $83,7^{a} \pm 2,5$ & $6,0^{b} \pm 2,0$ & $10,3^{b} \pm 2,5$ & $81,0^{\mathrm{a}} \pm 5,1$ & $7,4^{\mathrm{a}} \pm 4,0$ & $11,7^{a} \pm 2,0$ & $81,0^{\mathrm{b}} \pm 4,3$ & $6,4^{b} \pm 3,0$ & $12,7^{\mathrm{b}} \pm 2,0$ \\
\hline 200 & $72,4^{\mathrm{b}} \pm 4,1$ & $16,0^{\mathrm{a}} \pm 5,2$ & $11,7^{b} \pm 3,0$ & $80,4^{a} \pm 2,5$ & $6,7^{a} \pm 1,5$ & $13,0^{a} \pm 1,0$ & $80,7 \mathrm{~b} \pm 1,5$ & $6,4^{b} \pm 1,5$ & $13,0^{\mathrm{b}} \pm 1,0$ \\
\hline 300 & $65,4^{\mathrm{b}} \pm 3,5$ & $13,7^{\mathrm{ab}} \pm 1,1$ & $21,0^{\mathrm{a}} \pm 4,5$ & $78,7^{a} \pm 3,5$ & $7,4^{a} \pm 3,2$ & $14,0^{\mathrm{a}} \pm 2,0$ & $65,7^{c} \pm 2,0$ & $14,4^{\mathrm{a}} \pm 2,3$ & $20,0^{a} \pm 1,7$ \\
\hline
\end{tabular}

*Volúmenes en $\mu \mathrm{L}$ de aceite esencial que fueron expuestos los granos de maíz. ${ }^{1}$ Los valores son el promedio de tres réplicas \pm desviación estándar. ${ }^{2}$ Letras diferentes en columna indican diferencia significativa $(\mathrm{p}<0,05)$. Análisis de datos obtenidos del paquete estadístico Infostat versión 2018.

Tabla 5. Análisis de varianza (ANOVA) para los porcentajes de prueba de germinación sometidos a los diferentes tratamientos con aceites esenciales

\begin{tabular}{lrrrrl}
\hline \multicolumn{1}{c}{ F. V. } & SC & gl & CM & \multicolumn{1}{c}{ F } & p-valor \\
\hline Modelo & 2748,69 & 9 & 305,41 & 70,21 & $<0,0001$ \\
Tratamientos & 1731,37 & 3 & 577,12 & 132,67 & $<0,0001$ \\
Concentraciones & 713,56 & 2 & 356,78 & 82,01 & $<0,0001$ \\
Tratamientos * Concentración & 303,78 & 4 & 75,90 & 17,46 & $<0,0001$ \\
Error & 191,41 & 44 & 4,35 & & \\
Total & 2940,09 & 53 & & & \\
\hline
\end{tabular}

Análisis de varianza para los porcentajes de germinación (Valores transformados). 


\section{Contenido de fenoles y flavonoides totales}

De acuerdo con los resultados obtenidos (Tabla 6), el contenido de fenoles y flavonoides totales en el aceite de orégano (94,68 $\mathrm{mgEAG} / \mathrm{g}$ y $0,27 \mathrm{mgEQ} / \mathrm{g}$ respectivamente) fue mayor que en el de niaouli y eucalipto. Nuestros resultados concuerdan con los reportados por Camacho et al. (2011). Por otra parte, se observó un mayor contenido de fenoles y flavonoides totales en los tratamientos de maíz con orégano (16,09 mg EAG/g y 0,18 mg EQ/g respectivamente) en un volumen de $300 \mu \mathrm{L}$. Según Oliveira et al. (2009), esto podría ser atribuido al timol y carvacrol que son los componentes fenólicos presentes en mayor cantidad en el aceite de orégano, considerados buenos antioxidantes.

Tabla 6. Cuantificación de los fenoles y flavonoides totales de los extractos secos de maíz y aceites esenciales por Folin-Ciocalteu y Método Colorimétrico ${ }^{1,2}$.

\begin{tabular}{|c|c|c|c|c|}
\hline Muestras & ${ }^{*}$ Vol. $(\mu \mathrm{L})$ & Concentración Inicial & Fenoles & Flavonoides \\
\hline & & Muestra & (g/mL) mgEAG/g & $\mathrm{mgEQ} / \mathrm{g}$ \\
\hline Control (Maíz) & 0 & 0,02 & $4,15^{a} \pm 0,40$ & $0,14^{\text {bcde }} \pm 0,00$ \\
\hline Maíz + Niaouli & 100 & 0,02 & $0,84^{\underline{a}} \pm 0,51$ & $0,05^{\mathrm{ab}} \pm 0,00$ \\
\hline Maíz + Niaouli & 200 & 0,02 & $1,24^{a} \pm 0,51$ & $0,07^{\mathrm{abc}} \pm 0,05$ \\
\hline Maíz + Niaouli & 300 & 0,02 & $1,40^{\mathrm{a}} \pm 0,35$ & $0,08^{\mathrm{abcd}} \pm 0,00$ \\
\hline Maíz + Eucalipto & 100 & 0,02 & $2,23^{a} \pm 0,10$ & 0,09 abcde $\pm 0,01$ \\
\hline Maíz + Eucalipto & 200 & 0,02 & $3,57^{a} \pm 0,33$ & $0,11^{\text {abcde }} \pm 0,00$ \\
\hline Maíz + Eucalipto & 300 & 0,02 & $4,60^{\mathrm{a}} \pm 1,30$ & $0,11^{\text {abcde }} \pm 0,01$ \\
\hline Maíz + Orégano & 100 & 0,02 & $8,07^{a b} \pm 2,32$ & $0,16^{\text {cde }} \pm 0,00$ \\
\hline Maíz + Orégano & 200 & 0,02 & $12,53^{b c} \pm 1,79$ & $0,17^{\text {def }} \pm 0,00$ \\
\hline Maíz + Orégano & 300 & 0,02 & $16,09^{c} \pm 2,35$ & $0,18^{\text {ef }} \pm 0,03$ \\
\hline Niaouli & - & 0,02 & $1,68^{\mathrm{a}} \pm 0,40$ & $0,03^{a} \pm 0,01$ \\
\hline Eucalipto & - & 0,02 & $2,72^{\underline{a}} \pm 1,40$ & $0,03^{\underline{a}} \pm 0,01$ \\
\hline Orégano & & 0,02 & $94,68^{\mathrm{d}} \pm 1,09$ & $0,27^{\mathrm{f}} \pm 0,05$ \\
\hline
\end{tabular}

Volúmenes en $\mu \mathrm{L}$ de aceites esenciales que fueron expuestos los granos de maíz. ${ }^{1}$ Los valores son el promedio de tres réplicas \pm desviación estándar. ${ }^{2}$ Letras diferentes en columna indican significativa $(p<0,05)$. Análisis de datos obtenidos del paquete estadístico Infostat versión 2018.

\section{Contenido de antioxidantes método de DPPH y ABTS}

Se observó que el porcentaje de inhibición por el método de DPPH del aceite de orégano y el extracto de maíz control (sin aplicación de aceite esencial) fueron mayores que las demás muestras analizadas siendo de 76,31 y 71,92\% respectivamente (Tabla 7), no observándose diferencias significativas entre ellos ( $p>0,05)$. Este mismo comportamiento se observó en las mediciones con ABTS, sin embargo, el porcentaje de inhibición fue mayor (aceite de orégano 93,73 y control 92,19\%) en comparación con DPPH. Respecto a los tratamientos del maíz expuestos a los diferentes aceites esenciales, se observó una disminución en el \% de inhibición contra el radical DPPH, probablemente al combinarse los compuestos del maíz con el aceite, pudieron presentar antagonismos que en consecuencia repercutieron en menor afinidad por el radical DPPH. Por el contrario, en ABTS las muestras de maíz 
con aceite de orégano y con niaouli presentaron un rango de 89 a 91,89 \% de inhibición de este radical. Este efecto antioxidante puede ser relacionado con la presencia de timol y carvacrol que tienen un grupo hidroxilo $(-\mathrm{OH})$ unido al anillo aromático, presentando un débil carácter ácido, por lo que pueden donar átomos de hidrógeno con un electrón desemparejado $\left(\mathrm{H}^{\bullet}\right)$, un radical que se estabiliza por las estructuras de resonancia resultante de la deslocalización de los electrones en la molécula (Lima y Cardoso 2013; Carvalho 2017). Generalmente los radicales libres son compuestos muy reactivos que causan daños celulares provocando enfermedades crónico-degenerativas, por lo tanto, si se tienen productos con alta capacidad antioxidante, el alimento adquiere mayor valor agregado por tener un beneficio a la salud al consumirlo, como en el caso de los aceites esenciales y el maíz. Según Acevedo et al. (2013) el timol representa el 67,51\% del aceite de orégano. Esto explicaría los resultados obtenidos en la presente investigación considerando que el aceite de orégano fue el que presentó el mayor \% de inhibición en los dos métodos utilizados DPPH y ABTS, así como en los fenoles y flavonoides totales en comparación con los demás tratamientos. Los resultados concuerdan con los de Muñoz-Acevedo et al. (2009), Cervato et al. (2000), Porras-Loaiza y López-Malo (2009) y Flores-Martínez et al. (2016).

Tabla 7. Porcentaje de inhibición de los extractos secos de maíz y aceites esenciales por DPPH y ABTS ${ }^{1,2}$.

\begin{tabular}{lcccc}
\hline Muestras & *Vol. $(\mu \mathrm{L})$ & $\begin{array}{c}\text { Concentración Inicial } \\
\text { Muestra }(\mathrm{g} / \mathrm{mL}) \%\end{array}$ & Inhibición DPPH & \% Inhibición ABTS \\
\hline Control (Maíz) & 0 & 0,02 & $71,92^{\mathrm{b}} \pm 9,97$ & $92,19^{\mathrm{d}} \pm 0,20$ \\
Maíz + Niaouli & 100 & 0,02 & $40,69^{\mathrm{a}} \pm 0,22$ & $89,36^{\mathrm{b}} \pm 0,60$ \\
Maíz + Niaouli & 200 & 0,02 & $40,84^{\mathrm{a}} \pm 0,16$ & $89,93^{\mathrm{bc}} \pm 0,14$ \\
Maíz + Niaouli & 300 & 0,02 & $40,96^{\mathrm{a}} \pm 8,25$ & $90,30^{\mathrm{bc}} \pm 0,14$ \\
Maíz + Eucalipto & 100 & 0,02 & $49,02^{\mathrm{ab}} \pm 1,88$ & $90,92^{\mathrm{bc}} \pm 0,64$ \\
Maíz + Eucalipto & 200 & 0,02 & $49,45^{\mathrm{ab}} \pm 1,03$ & $91,17^{\mathrm{bc}} \pm 0,71$ \\
Maíz + Eucalipto & 300 & 0,02 & $49,57^{\mathrm{ab}} \pm 3,48$ & $91,57^{\mathrm{c}} \pm 0,30$ \\
Maíz + Orégano & 100 & 0,02 & $52,26^{\mathrm{ab}} \pm 0,66$ & $91,65^{\mathrm{cd}} \pm 0,06$ \\
Maíz + Orégano & 200 & 0,02 & $56,59^{\mathrm{ab}} \pm 4,22$ & $91,75^{\mathrm{cd}} \pm 0,33$ \\
Maíz + Orégano & 300 & 0,02 & $60,71^{\mathrm{ab}} \pm 6,00$ & $91,899^{\mathrm{cd}} \pm 0,24$ \\
Niaouli & - & 0,02 & $34,98^{\mathrm{a}} \pm 0,48$ & $35,21^{\mathrm{a}} \pm 1,72$ \\
Eucalipto & - & 0,02 & $35,71^{\mathrm{a}} \pm 2,56$ & $36,80^{\mathrm{a}} \pm 0,99$ \\
Orégano & - & 0,02 & $76,31^{\mathrm{b}} \pm 1,64$ & $93,73^{\mathrm{d}} \pm 0,39$ \\
\hline
\end{tabular}

Volúmenes en $\mu \mathrm{L}$ de aceites esenciales que fueron expuestos los granos de maíz. ${ }^{1}$ Los valores son el promedio de tres réplicas \pm desviación estándar. ${ }^{2}$ Letras diferentes en columna indican diferencia significativa $(\mathrm{p}<0,05)$. Análisis de datos obtenidos del paquete estadístico Infostat versión 2018.

\section{Conclusiones}

Los aceites de orégano, niaouli y eucalipto mostraron tener biocontrol del insecto $S$. zeamais pudiendo ser utilizados para el control de plagas en productos poscosecha, en este caso el maíz, como una alternativa natural para reducir la presencia de insectos durante el almacenamiento. Por otra parte, la capacidad antioxidante proporcionada por el aceite de orégano, el maíz y sus combinaciones, confieren al producto mayor valor agregado al ofrecer a los consumidores productos que puedan evitar o prevenir enfermedades crónicodegenerativas al consumirlos inhibiendo a los radicales libres causantes de daños celulares, obteniendo así beneficios a la salud. 


\section{Agradecimientos}

Los autores agradecen al programa de proyectos internos financiados por la Universidad de Sonora Clave (USO313006038) para realizar la investigación. Al CONACyT por la beca otorgada a Francyelli Regina Costa Becheleni para la realización de sus estudios de posgrado.

\section{Literatura Citada}

Abbott, W.S. (1925) A method of computing the effectiveness of an insecticide. Journal of Economic Entomology, 18(2): 265-267. https: / / doi.org/10.1093/jee/18.2.265a

Acevedo, D., Navarro, M. y Monroy, L. (2013) Composición química del aceite esencial de hojas de orégano (Origanum vulgare). Información Tecnológica, 24(4): 43-48. http:// dx.doi.org / 10.4067 / S0718-07642013000400005

Antunes, L. y Dionello, R.G. (2017) Efficacy of insetitis during the burning of milky stones. The Scientific Electronic Journal of UERGS, 3(1): 83-94. https://doi. org/0479.10.21674/2448-31.83-94

Assis, C., Almeida, F., Silva, J., Queiroga, V., Figueiredo, N., Cárdenas, O. y Rojas, A. (2014) Eficiencia vegetales como insecticida sobre Sitophilus zeamais en grano de maíz almacenado. Revista Ciencias Técnicas Agropecuarias, 23(2): 57-62.

Blanco, V., Durañona, H. y Acosta-Roca, R. (2016) Efecto de la temperatura y la humedad en la conservación de granos de maíz en silos metálicos refrigerados. Cultivos Tropicales, 37(4): 105-114. http: / / dx.doi.org/10.13140/RG.2.2.13900.21127

Bustos, G., Silva, G., Fisher, S., Figueroa, I., Urbina, A. y Rodríguez, J.C. (2017) Repelencia de mezclas de aceites esenciales de boldo, laurel chileno y tepa contra el gorgojo del maíz. Southwestern Entomologist, 42(2): 551-563. http://dx.doi. org / 10.3958/059.042.0224

Çalmaşur, Ö., Aslan, İ. y Şahin, F. (2006) Insecticidal and acaricidal effect of three Lamiaceae plant essential oils against Tetranychus urticae Koch and Bemisia tabaci Genn. Industrial Crops and Products, 23(2): 140-146. http:/ / dx.doi.org/10.1016/j.indcrop.2005.05.003

Camacho, D., López, I., Sojo, C., Linares, O., Martínez, C.M. y Pantaleón, D.G. (2011) Efecto de extractos de oregano (Origanum vulgare L.) ante la oxidación en aceite vegetal comestible. Revista de la Facultad de Agronomía, 28(1): 628-635.

Campanini, E., Davolos, C., Alves, E. y Lemos, M. (2012) Caracterização de novos isolados de Bacillus thuringiensis para o controle de importantes insetos-praga da agricultura. Bragantia, 71(3): 362-369. http://dx.doi.org/10.1590/S000687052012000300007

Carvalho, A., Lopes, A., Rezende, C.N., Carneiro, L., Meirelles, V., Lara, V. y Lorini, I. (2017) Manejo integrado de pragas de grãos armazenados: implantação e monitoramento de pragas na unidade armazenadora. Embrapa Soja-Artigo em Periódica Indexado (ALICE), 13(17): 36-61.

Cervato, G., Carabelli, M., Gervasio, S., Cittera, A., Cazzola, R. y Cestaro, B. (2000) Antioxbdant properties of oregano (Origanum vulgare) leaf extracts. Journal of Food Biochemistry, 24(6): 453-465. https: / / doi.org/10.1111/j.1745-4514.2000.tb00715.x

Docampo, P.C., Spera, M. y Voitzuk, A.P. (2014) Serie de casos de intoxicación fatal por ingesta intencional de fosfuro de aluminio. estrategias de prevención secundaria. Acta Toxicológica Argentina, 22(3): 23-26.

Eldeen, I., Seow, E., Abdullah, R. y Sulaiman, S. (2011) In vitro antibacterial, antioxidant, total phenolic contents and anti-HIV-1 reverse transcriptase activities of extracts of seven Phyllanthus sp. South African Journal of Botany, 77(1): 75-79. https:// doi. org/10.1016/j.sajb.2010.05.009 
Elias, M.C., Lopes, V., Gutkoski, L.C., Oliveira, M., Mazzutti, S. y Guerra Dias, A.R. (2009) Umidade de colheita, métodos de secagem e tempo de armazenamento na qualidade tecnológica de grãos de trigo (cv.'Embrapa 16'). Ciência Rural, 39(1): 25-30. https:/ / doi. org / 10.1590/S0103-84782009000100005

FAO (Food and Agriculture Organization, Forestry Department) (2010) Seeds in Emergencies: A technical handbook. FAO Plant Production and Protection Paper. p. 7688.

Finkler, C. (2011) Controle de insetos: uma breve revisão. Anais da Academia Pernambuca na de Ciência Agronômica. Revista Científica Agraria, 8(9): 169-189. http://dx.doi. org/10.4322/ rca.2217

Florão, A., Fonseca, R., López, M. y Gabriel, M. (2004) FOSFINA: RISCOS. Visão Acadêmica, 5(2): 101-118.

Flores, M., León, C., Estarrón E. y Orozco, A. (2016) Optimización del proceso de extracción de sustancias antioxidantes a partir del orégano mexicano (Lippia graveolenshbk) utilizando la metodología de superficie de respuesta (msr). Revista Mexicana de Ingeniería Química, 15(3): 773-785.

González, A. (1995) El maíz y su conservación, México. Editorial Trillas, México D.F. 365399.

González-Guiñez, R., Silva-Aguayo, G., Urbina-Parra, A. y Gerding-González, M. (2016) Aceite esencial de Eucalyptus globulus Labill y Eucalyptus nitens H. Deane \& Maiden (MYRTACEAE) para el control de Sitophilus zeamais Motschulsky. Chilean Journal of Agricultural and Animal Sciences, 32(3): 204-216. http://dx.doi.org/10.4067/S071938902016005000005

Gorham, J. (1999) Insect and mite pests in food. An illustrated key. National Agricultural Library USDA, 1(2): 228-229.

ISTA (International Seed Testing Association) (2016) Disponible en: http: / / www.seedtest. org

InfoStat (2018) Software estadístico desarrollado por Universidad Nacional de Córdoba (FCA-UNC).Disponibleen:https:/ / www.infostat.com.ar/index.php?mod=page\&id=34

Langsi, D.J., Tofel, H.K., Fokunang, C.N., Suh, C., Eloh, K., Caboni, P. y Nukenine, E.N. (2018) Insecticidal activity of essential oils of Chenopodium ambrosioides L. and Cupressuss sempervirens L. ad their binary combinations on Sitophilus zeamais. GSC Biological and Pharmaceutical Sciences, 3(2): 24-34.

Lagunes, A. (1994) Uso de extractos, polvos vegetales, y polvos minerales para el combate de plagas del maíz y del frijol en la agricultura de subsistencia. Colegio de Postgraduados. USAID, CONACYT, BORUCONSA. Texcoco, México. 35 pp.

Lima, R. y Cardoso, M. (2013) Família Lamiaceae: Importantes óleos essenciais com ação biológica e oxidante. Revista Fitos, 3(3): 14-24.

Manach, C., Scalbert, A., Morand, C., Rémésy, C. y Jiménez, L. (2004) Polyphenols: food sources and bioavailability. American Journal of Clinical Nutrition, 79: 727-747.

Martínez, E., Borboa, F., Wong, C., Cinco, M., Del Toro, S., Ortega, N., Cornejo, R., Ozuna, A. y Cabral, T. (2015) Insecticidal efficacy and repellency of oregano (Lippia palmeri) essential oil for control of Sitophilus zeamais and Prostephanus truncatus in stored maize. South Western Entomology, 40(4): 713-720. https: / / doi.org/10.3958/059.040.0410

Mazzonetto, F. (2002) Efeito de genótipos de feijoeiro e de pós de origem vegetal sobre Zabrotes subfasciatus (Boh.) e Acanthoscelides obtectus (Say) (Col.: Bruchidae) (Doctoral dissertation, Universidade de São Paulo). http://dx.doi.org/10.11606/T.11.2002.tde31072002-141929

Molyneux, P. (2004) The use of the stable free radicaldiphenylpicrilhydrayl (DPPH) for estimating antioxidant activity, Songklanakarin. Journal of Science and Technology, 26(2): 211-219. 
Morales, D., Gutiérrez, L., Robles, G., Aguilar, J., Lugo, C., Guerrero M. y Del Toro, S. (2015) Anti-inflammatory activity and changes in antioxidant properties of leaf and stem extracts from Vitex mollis kunth during in vitro digestion. Evidence-Based Complementary and Alternative Medicine, 2015. http:/ / dx.doi.org/10.1155/2015/349235

Muñoz-Acevedo, A., Kouznetsov, V.V. y Stashenko, E.E. (2009) Composición y capacidad antioxidante in-vitro de aceites esenciales ricos en Timol, Carvacrol, trans-Anetol o Estragol. Revista de la Universidad Santander. Salud UIS, 41(3): 287-294.

Norambuena, C., Silva, G., Urbina, A., Figueroa, I. y Rodríguez, M. (2016) Insecticidal activity of Laureliopsis philippiana (Looser) Schodde (Atherospermataceae) essential oil against Sitophilus spp. (Coleoptera Curculionidae). Chilean. Journal of Agricultural Research, 76(3): 330-336. http: / / dx.doi.org/10.4067/S0718-58392016000300010

Ogendo, O., Kostyukovsky, M., Ravid, U., Matasyoh, J., Deng, A., Omolo, E., Kariuki, S. y Shaaya, E. (2008) Bioactivity of Ocimum gratissimum L. and two of its constituents against five insect pests attacking stored food products. Journal of Stored Producs Research, 44: 328-334.

Oliveira, R., Reis, T., Sacramento, C., Duarte, L. y Oliveira, F. (2009) Constituintes químicos voláteis de especiarias ricas em eugenol. Revista Brasileira de Farmacognosia, 19(3): 771775. https: / / doi.org / 10.1590/S0102-695X2009000500020

Ortiz, R., Ramírez, A., González, E. y Velázquez, M. (2015) Almacenes de maíz en México: Tipología y Caracterización. Estudios Sociales, 23(45): 163-184.

Ortiz, U., Silva, A., Urbina, P., Zapata, S.M., Rodríguez, M., Concepción, J. y Lagunes, T. (2012) Bioactividad del polvo de tepa (Laureliopsis philippiana (Looser) Shodde) para el control de Sitophilus zeamais Motschulsky en Laboratorio. Chilean Journal of Agricultural Research, 72(1): 68-73. http: / dx.doi.org/10.4067/S0718-58392012000100011

Pessoa, A., Lozano, E., Vilani, A., Potrich, M., Matos, L., Oliveira, T. y Pessoa, G.M. (2014) Bacillus thuringiensis Berliner and Anticarsia gemmatalis Hübner (Lepidoptera: Erebidae) under the action of plant extracts. Arquivos do Instituto Biológico, 81(4): 329-334. http: / / dx.doi.org / 10.1590/1808-1657000962012

Porras, L. y López, M. (2009) Importancia de los grupos fenólicos en los alimentos. Temas Selectos de Ingeniería de Alimentos, 3(1): 121-134.

Reyes-Guzmán, R., Borboa-Flores, J., Cinco-Moroyoqui, F.J., Rosas-Burgos, E.C., Osuna-Amarillas, P.S., A., Wong-Corral, F.J., Ortega-Nieblas, M.M. y León-Lara, J.D. (2012) Actividad insecticida de aceites esenciales de dos especies de Eucalyptus sobre Rhyzopertha dominica y su efecto en enzimas digestivas de progenies. Revista Chapingo Serie Ciencias Forestales y del Ambiente, 18(3): 385-394. https://doi.org/10.5154/r. rchscfa.2012.02.015

Rees, P. (1996) Coleoptera. In: Subramanyam, B. and Hagstrum, D. (eds.). Integrated management of insects in stored products. Marcel Dekker, New York, USA. p. 1-40.

Salas, J. (1985) Protección de semillas de maíz (Zea mays) contra el ataque de Sitophilus oryzae a través del uso de aceites vegetales. Agronomía Tropical, 35(4-6): 13-18.

SAG (Servicio Agrícola y Ganadero de Chile) (2000) Normas generales y específicas de certificación de semillas. Ministerio de Agricultura, Chile. Disponible en http: / / www. sag.gob.cl/sites / default/files / NORMAS_GENERALES_SEMILLAS.pdf

Santos, W. y Chavaglia, R. (2017) Importância do controle de armazenagem para conservação e comercialização de grãos. Revista Científica do Centro de Ensino Superior, 5(5): 155.

Silva, A., Kiger, M., Hepp, G. y Tapia, V. (2005) Control de Sitophilus zeamais con polvos vegetales de tres especies del género Chenopodium. Pesquisa Agropecuária Brasileira, 40(10): 953-960.

Usano, A., Paúl, S. y Días, S. (2014) Aceites esenciales: conceptos básicos y actividad antibacteriana. Reduca (Biología), Serie Botanica, 7(2): 60-70. 
Venu, P., Holm, D. y Jayanty, S. (2012) Effects of cooking methods on polyphenols, pigments and antioxidant activity in potato tubers. LWT-Food Science and Technology, 45(2): 161171. http: / / dx.doi.org/10.1016/j.lwt.2011.08.005 